\title{
Competitive mechanisms of the functioning of social and economic systems
}

\author{
Vera Poryadina, ${ }^{1, *}$, Vladimir Burkov ${ }^{1}$, Sergey Barkalov ${ }^{1}$ \\ ${ }^{1}$ Voronezh State Technical University, Moscow Avenue, 14, Voronezh, 394026, Russia
}

\begin{abstract}
To increase the efficiency of production in a planned economy, it is necessary to develop mechanisms of functioning that encourage enterprises to maximize the accounting of all reserves for increasing production efficiency. In this article, a model of a competitive mechanism for the functioning of active systems is developed. A feature of competitive mechanisms is that planning or incentive procedures include a competition procedure between active elements claiming to receive a profitable plan or any preferential terms of resource provision, financing and incentives. The authors conducted a study of the competitive mechanism on the example of the problem of resource allocation; It is proved that there is a Nash equilibrium for the competitive mechanism. The paper formulated and proved a theorem on the optimality of competitive mechanisms, which shows how effective the competitive mechanism for the distribution of limited resources.
\end{abstract}

\section{Introduction}

To increase the efficiency of production in a planned economy, it is necessary to develop mechanisms of functioning that induce enterprises (organizations) to maximum allowance for all reserves for improving production efficiency both at the stage of plan development and at the stages of its implementation [1,2]. The so-called competitive mechanisms attract great attention to the theory and practice of management for solving this problem. A feature of competitive mechanisms is that planning or incentive procedures include a competition procedure between active elements claiming to receive a profitable plan or any preferential terms of resource provision, financing and incentives.

\section{Model of the competitive resource allocation mechanism}

Let, for example, we are talking about the distribution of capital investments among the enterprises of the industry. Each enterprise receives a certain base value of capital investments. Denote this base value by. To receive more funds, the enterprise must evaluate the effect of using these funds [3, 4]. The distribution of capital investments on a competitive basis means that, first of all, additional funds (more than the base amount c) are received by enterprises that have a high efficiency of using capital investments. The

\footnotetext{
*Corresponding author: poryadina08@mail.ru
} 
concept of resource use efficiency is as follows: if the effect $\varphi_{i}\left(x_{i}\right)$, that an enterprise expects to receive from the use of a resource in quantity is determined $x_{i}$, then the efficiency $\xi_{i}=\varphi_{i}\left(x_{i}\right) / x_{i}$ is equal to the effect per unit of resource (per 1 rub of capital investment).

In the competitive mechanism, the principle of resource allocation is another - the resource in the required quantity is received only by the winners of the competition. If the number of winners is equal $\mathrm{m}$ and given, then the winners are the $\mathrm{m}$-th companies with the highest efficiency ratings (the rest get the base value of the resource $c$ ). So, each enterprise - the participant of competition - informs to organizers of competition two sizes: the funds $\mathrm{s}_{\mathrm{i}}$, equired to carry out the measure to improve the efficiency of production, and an assessment $\xi_{i}$ f the expected effectiveness of these activities. This can be the development of new technology, the reconstruction of the enterprise, the construction of a new workshop, etc. The expected effect $\omega_{i}$ of the $i$-th enterprise project is defined as the product of efficiency by the amount of the required means, i.e. $\omega_{i}=\xi_{i} \cdot s_{i}$. We assign enterprises numbers in descending order $\xi_{i}$, so that $\xi_{1} \geq \xi_{2} \geq \Lambda \geq \xi_{m} \geq \xi_{m+1} \geq \Lambda \geq \xi_{n}$.

Winners are the first $m$ companies. In case, if $\xi_{m}=\xi_{m+1}$, the winners are determined ambiguously (as a rule, then additional criteria are used for unambiguous choice). Further, the winners should undertake the implementation of the planned event and give the promised effect in the form of new quality products, a new modern technology or a new workshop. There may be doubts about the effectiveness of such a mechanism: first the enterprise promises a high result, becomes a winner, receives funds, and then time passes, there are "objective reasons" for failure. Therefore, for the effectiveness of competitive mechanisms, an effective monitoring system is necessary (for projects that require a lot of time, there must be a phased control system). Note that the real magnitude of the effect, which the head really expects to receive, if he is allocated funds in the amount of $\mathrm{s}_{\mathrm{i}}$, is $\varphi_{i}\left(s_{i}\right)$. The difference $\xi_{i} s_{i}-\varphi_{i}\left(s_{i}\right)$ determines the amount of deliberate overstatement of the effect, in other words, the fraud to which the leader can consciously go in order to win the tender and get the funds necessary for the development of the enterprise. The effectiveness of the control system will be determined by the amount of penalties $\chi_{i}=\alpha\left(\xi_{i} s_{i}-\varphi_{i}\left(s_{i}\right)\right), \alpha>0$, if the promised effect is not achieved, i.e., if the effect $\varphi_{i}\left(s_{i}\right)$ is less than the planned effect $\xi_{i} s_{i}$.

We describe the system of incentives for enterprises, encouraging them to participate in the competition. We assume that part of the economic effect remains with the enterprise. If we designate this part through $\mu<1$, then the target function of the enterprise can be written in the form:

$$
f_{i}\left(\varphi_{i}, \xi_{i}\right)=\mu \varphi_{i}\left(s_{i}\right)-\alpha\left[\xi_{i} s_{i}-\varphi_{i}\left(s_{i}\right)\right]
$$

Here all the components are taken into account: the part of the effect that the enterprise receives and the penalties it pays. It should be borne in mind that fines are calculated only if $\xi_{i} s_{i}>\varphi_{i}\left(s_{i}\right)$. Otherwise, the penalties are equal $\chi_{i}=0$, and the objective function is $\mu \varphi_{i}\left(s_{i}\right)$.

\section{Analysis of the competitive mechanism}

To analyze the competitive mechanism means to answer three questions: 
1. Who will be the winner of the competition?

2. What is the effectiveness of each enterprise?

3. How many resources will each winner receive?

We will answer these questions in the reverse order. Note that the victory in the contest depends only on the evaluation of efficiency $\xi_{i}$ and does not depend on the size of the resource $s_{i}$ being ordered. Therefore, of course, the enterprise will order the amount of the resource such that, in case of its victory, the value of the objective function was maximum at the stated efficiency $\xi_{i}$.

We assume that $\varphi_{i}\left(s_{i}\right)$ - strictly concave differentiable nondecreasing functions $s_{i}, \varphi_{i}(0)=0$.

Then the optimal claim $S_{i}$ is determined from the condition

$$
\partial \chi_{i} \in \mu \frac{d \mu_{i}\left(s_{i}\right)}{d s_{i}}
$$

if, of course, this condition has a positive solution. Otherwise, the optimal bid $s_{i}=0$. In (2) the sign $\partial$ denotes the subdifferential.

If conditions (2) are satisfied at a point $s_{i}=\theta_{i}^{c}\left(\xi_{i}\right)$, that is a solution of equation

$$
s_{i} \xi_{i}=\varphi_{i}\left(s_{i}\right)
$$

then we will say that for $i$-th enterprise there are "strong" penalties. If condition (2) is satisfied at the point $s_{i}=\theta_{i}\left(\xi_{i}\right)$, such that $\xi_{i} \theta_{i}\left(\xi_{i}\right)>\varphi_{i}\left[\theta_{i}\left(\xi_{i}\right)\right]$, then condition (2) takes the form:

$$
\frac{d \varphi_{i}\left(s_{i}\right)}{d s_{i}}=\frac{\alpha \xi_{i}}{\alpha+\mu}
$$

Combining two cases: (3) and (4), the optimal claim can be represented in the form: $s_{i}^{O P T}\left(\xi_{i}\right)=\max \left[\theta_{i}\left(\xi_{i}\right), \theta_{i}^{c}\left(\xi_{i}\right)\right]$.

Further we will consider only two situations: either for all enterprises there are "strong fines", i.e. applications are determined by the condition (3), or "weak fines", i.e. application is determined by the condition (4). Intermediate cases where for one part of the winning enterprises the application is determined by the condition of "strong fine", for the other part - by the condition of "weak fines", they are excluded from consideration. Subsequent theorems on competitive resource allocation mechanisms will only be valid for two cases $[5,6]$.

Thus, if an enterprise was among the winners of the competition, having reported an evaluation of the effectiveness $\xi_{i}$, then the effect of using the funds received $s_{i}^{O P T}\left(\xi_{i}\right)$ with allowance for fines will be $\mu \varphi_{i}\left(s_{i}^{O P T}\left(\xi_{i}\right)\right)-\alpha\left[\xi_{i} s_{i}^{O P T}\left(\xi_{i}\right)-\varphi_{i}\left(s_{i}^{\mathrm{OPT}}\left(\xi_{i}\right)\right)\right]$

We denote this quantity by $h_{i}\left(\xi_{i}\right)$.

Lemma 1. a) The function $h_{i}\left(\xi_{i}\right)$ is continuous, decreasing in $\xi_{i}$; b) $\sup _{0<\xi_{i}<\infty} h_{i}\left(\xi_{i}\right) \geq \mu \varphi_{i}(c) ;$ c) $\lim _{\xi_{i} \rightarrow \infty} h_{i}\left(\xi_{i}\right)=0$.

Evidence. a) Consider the function $h(\xi)=\alpha \max _{x}\left[\frac{\mu+\alpha}{\alpha} \varphi(x)-\xi x\right]$, where $\varphi(x)-$ 
strictly concave differentiable for $x>0$ function, $\varphi(0)=0$. Let the function $h(\xi)$ be continuous at this point $\xi=\xi^{0}$. Let us show that $h(\xi)$ it is continuous at this point.

Because of the differentiability and strict concavity of the function $\frac{\mu+\alpha}{\alpha} \varphi(x)-\xi^{0} x$ it attains its maximum by $x$ at the unique point $x^{0}$, determined by the condition $\frac{\mu+\alpha}{\alpha} \varphi^{\prime}\left(x^{0}\right)=\xi^{0}$. From the strict concavity of the function $\frac{\mu+\alpha}{\alpha} \varphi(x)-\xi x$ by $x$ follows that $\frac{\mu+\alpha}{\alpha} \varphi^{\prime}(x)-\xi$ decreases by $x$. Thus, for a fixed $\xi \frac{\mu+\alpha}{\alpha} \varphi(x)-\xi x$ is a function of one variable $x$, then its derivative has no discontinuities of the first kind, but from the boundedness and monotonicity of this derivative on $\left[\varepsilon^{\prime}, \infty\right)$, where $\varepsilon^{\prime}>0$, follows that $\frac{\mu+\alpha}{\alpha} \varphi^{\prime}(x)-\xi$ has no discontinuities of the second kind. In this way, $\frac{\mu+\alpha}{\alpha} \varphi^{\prime}(x)-\xi-$ continuous, decreasing function.

It follows from the foregoing that the equation $\frac{\mu+\alpha}{\alpha} \varphi^{\prime}(x)-\xi^{0}-\delta=0$ for a value sufficiently small in absolute value $\delta$ has a unique solution $x_{\delta}$. Wherein $\left|x_{\delta}-x^{0}\right|<v_{\delta}$, where $\lim _{\delta \rightarrow 0} v_{\delta}=0$. Really, if $\lim _{\delta \rightarrow 0} v_{\delta}=$ const $>0$, then we get that the maximum of the function $\frac{\mu+\alpha}{\alpha} \varphi(\chi)-\xi^{0} \chi$ is attained at least in two different points, and this contradicts the strict concavity of the function $\frac{\mu+\alpha}{\alpha} \varphi(x)-\xi^{0} x$.

Let $\xi=\xi^{0}+\delta$. We show that for $\forall \varepsilon>0, \exists \delta_{\varepsilon}>0$, such that for $\forall \delta<\delta_{\varepsilon}$, if $\left|\xi-\xi^{0}\right|<\delta,\left|h(\xi)-h\left(\xi^{0}\right)\right|<\varepsilon, h(\xi)-$ continuous.

Consider

$$
\begin{aligned}
& \quad\left|h(\xi)-h\left(\xi^{0}\right)\right|=\alpha\left|\max _{x}\left(\frac{1+\alpha}{\alpha} \varphi(x)-\xi^{0} x-\delta x\right)-\max _{\chi}\left(\frac{1+\alpha}{\alpha} \varphi(\chi)-\xi^{0} \chi\right)\right|= \\
& =\alpha\left|\frac{1+\alpha}{\alpha} \varphi\left(x_{\delta}\right)-\xi^{0} x_{\delta}-\delta x-\frac{1+\alpha}{\alpha} \varphi\left(x^{0}\right)-\xi^{0} x^{0}\right| \leq(1+\alpha)\left|\varphi\left(x^{0}\right)-\varphi\left(x_{\delta}\right)\right|+ \\
& \quad+\alpha \xi^{0}\left|x_{\delta}-x^{0}\right|+\alpha \delta x_{\delta} \leq\left[(1+\alpha)\left|\varphi\left(x^{\prime}\right)+\alpha \xi^{0}+\alpha \delta\right|\right] y_{\delta}+\alpha \delta x^{0}, \\
& \text { where } x^{\prime} \in\left[\chi_{\delta}, \chi^{0}\right] .
\end{aligned}
$$

Since the quantities $\left|\varphi\left(x^{\prime}\right)\right|$ and $\xi^{0}$ are bounded, and $\lim _{\delta \rightarrow 0} v_{\delta}=0$, it can be chosen $\delta$ so small that $\left[(1+\alpha)\left|\varphi\left(x^{\prime}\right)\right|+\alpha \xi^{0}+\alpha \delta\right] v_{\delta}+\alpha x^{0} \delta<\varepsilon$.

The case $\xi=\xi^{0}-\delta$ is treated similarly. Thus, the function $h(\xi)$ is continuous in the case of weak penalties.

For strong penalties, the extremum point of a function $\varphi_{i}$ is determined by the condition (3), which is solvable with respect to $x_{i}$, if $\varphi_{i}^{\prime}(0)>\xi_{i}$, otherwise $x_{i}=0$. This follows from the strict concavity of the function $\varphi_{i}\left(x_{i}\right)$. Therefore, there is $0<\varphi^{\prime}\left(x_{i}\right)<\xi_{i}$ at the point $x_{i}$. But then, by the implicit theorem, the function $x_{i}\left(\xi_{i}\right)$ is continuously differentiable. Thus, the function $h_{i}\left(\xi_{i}\right)$ is continuous and in the case of "strong penalties". 
We now show that $h_{i}\left(\xi_{i}\right)$-decreasing function.

We first consider the case of "weak fines" when $h_{i}\left(\xi_{i}\right)=(\mu+\alpha) \varphi_{i}\left(x\left(\xi_{i}\right)\right)-\alpha \xi_{i} x\left(\xi_{i}\right)$.

Let $\xi_{i}^{2}>\xi_{i}^{1}$, then takes place $h_{i}\left(\xi_{i}^{\prime}\right)=(\mu+\alpha) \varphi_{i}\left(x_{i}^{\prime}\right)-\alpha \xi_{i}^{\prime} x_{i}^{\prime} \geq(\mu+\alpha) \varphi_{i}\left(x_{i}^{2}\right)-\alpha \xi_{i}^{\prime}>(\mu+\alpha) \varphi_{i}\left(x_{i}^{2}\right)-\alpha \xi_{i}^{2} x_{i}^{2}=h_{i}\left(\xi_{i}^{2}\right)$ i. e. $h_{i}\left(\xi_{i}\right)$ - decreasing function.

Let now there are "strong fines". In this case $h_{i}\left(\xi_{i}\right)=\mu \varphi_{i}\left(x_{i}\left(\xi_{i}\right)\right)$, where $x_{i}\left(\xi_{i}\right)$ satisfy (3). We consider the derivative of the function $h_{i}\left(\xi_{i}\right): h_{i}^{\prime}\left(\xi_{i}\right)=\mu \varphi_{i}^{\prime}\left(x_{i}\left(\xi_{i}\right)\right) x_{i}^{\prime}\left(\xi_{i}\right)$. Here $\varphi_{i}^{\prime}\left(x_{i}\left(\xi_{i}^{\prime}\right)\right)>0$. Let's study the sign $x_{i}^{\prime}\left(\xi_{i}\right)$. Differentiating the function $\varphi_{i}\left(x_{i}\left(\xi_{i}\right)\right)-x_{i}\left(\xi_{i}\right) \xi_{i}$ by $\xi_{i}$ and equating the derivative of this function to zero, we obtain:

$$
\left[\varphi^{\prime}\left(x_{i}\right)-\xi_{i}\right] x_{i}^{\prime}\left(\xi_{i}\right)=x_{i}\left(\xi_{i}\right) .
$$

As $x_{i}\left(\xi_{i}\right)>0$, and $\varphi^{\prime}\left(x_{i}\left(\xi_{i}\right)\right)-\xi_{i}<0$, then $x_{i}^{\prime}\left(\xi_{i}\right)<0$. From here $h_{i}^{\prime}\left(\xi_{i}\right)<0$.

b) The following chain of inequalities holds:

$$
\sup _{0<\xi_{i}<\infty} h_{i}\left(\xi_{i}\right) \geq h_{i}(0)=\sup _{s_{i}} \mu \varphi_{i}\left(s_{i}\right) \geq \mu \varphi_{i}(c)^{\circ}
$$

c) We show that $\lim _{\xi_{i} \rightarrow \infty} x_{i}\left(\xi_{i}\right)=0$. Let $\lim _{\xi_{i} \rightarrow \infty} x_{i}\left(\xi_{i}\right)=c_{i}^{0}>0$, then $\varphi_{i}\left(\chi_{i}\right) \rightarrow \infty$ at $\chi_{i} \rightarrow c_{i}^{0}$, which contradicts the boundedness of the derivative $\varphi_{i}^{\prime}$ on $(\varepsilon, \infty)$, where $\varepsilon>0$. It is easy to show that from $\lim _{\xi_{i} \rightarrow \infty} \chi_{i}\left(\xi_{i}\right)=0$ explores $\lim _{\xi_{i} \rightarrow \infty} h_{i}\left(\xi_{i}\right)=0$ at $\xi_{i} \rightarrow \infty$. The lemma is proved.

Let's notice now that the enterprise which has not entered into number of winners of competition, receives resources in quantity $c_{i}$, this provides him with an effect $\Delta_{i}=\mu \varphi_{i}\left(c_{i}\right)$. It is obvious that for the interest of enterprises to participate in the contest it is necessary that the effect, taking into account the fines in case of victory in the competition, be at least $\Delta_{i}, h_{i}\left(\xi_{i}\right) \geq \Delta_{i}$. We denote by $v_{i}$ solution of equation $h_{i}\left(v_{i}\right)=\Delta_{i}$ (the corresponding conditions for the existence of the solution are assumed to be satisfied). The value $v_{i}$ determines the maximum efficiency that the enterprise will report. Let enterprises be sorted in descending order $v_{i}, v_{1} \geq v_{2} \geq \Lambda \geq v_{n}$. With the number of winners of the competition equal $\mathrm{m}$, to the first $\mathrm{m}$ enterprises it is enough to inform the efficiency evaluations $v_{m+1} \leq \xi_{i} \leq v_{i}, i=1,2, \ldots, m$, in order to enter the number of winners (in case if $\xi_{i}=v_{m+1}$, we assume that the enterprise $i$ becomes a winner as a result of taking into account additional criteria). So, a lot of winners are defined - these $m$ enterprises with the largest values $v_{i}$. Since the effect is subject to penalties $h_{i}(\xi)$-decreasing function $\xi_{i}$, then, of course, the winners will report the minimum performance scores that still allow them to remain among the winners, i.e. assessments $s_{i}^{*}=v_{m+1}$. Thus, we have proved the following result.

Theorem 1. For a competitive mechanism, there is a Nash equilibrium (Nash point), and for any Nash point there is:

$$
\xi_{i}^{*}=v_{m+1}, i \in Q
$$

where $\mathrm{Q}-\mathrm{a}$ lot of winners of the competition (a lot of $m$ enterprises with the greatest 
values $v_{i}$ ).

You can imagine the following scenario of the competition. First, all participants report performance ratings $\xi_{i}=v_{i}$. This, it can be said, is a preliminary stage in identifying the "strength" of the applicants.

Immediately identify the winners who reported the highest ratings, as well as the "strength" $v_{m+1}$ of the enterprise closest to the winners. At the second stage, the winners refine the efficiency estimates (reduce them to $\xi_{i}^{*}=v_{m+1}$ ), while increasing the application for the resource.

Example 1. Let $\varphi_{i}\left(x_{i}\right)=2 \sqrt{r_{i} x_{i}}, i=1, \ldots, n$. Define the optimal application for the resource for the winners in the case of weak penalties:

$$
\begin{aligned}
& \frac{d \varphi_{i}}{d s_{i}}=\sqrt{\frac{r_{i}}{s_{i}}}=\frac{\alpha \xi_{i}}{\alpha+\mu}, \\
& \theta_{i}\left(\xi_{i}\right)=r_{i}\left(\frac{\alpha+\mu}{\alpha \xi_{i}}\right)^{2} .
\end{aligned}
$$

In the case of heavy fines, the optimal bid is determined from equation

$$
2 \sqrt{r_{i} s_{i}}=\xi_{i} s_{i}, \theta_{i}^{c}\left(\xi_{i}\right)=\frac{4 r_{i}}{\xi_{i}^{2}} .
$$

The case of a weak penalty is determined by the condition $\theta_{i}\left(\xi_{i}\right)>\theta_{i}^{c}\left(\xi_{i}\right)$ or

$$
\frac{\alpha+\mu}{\alpha \xi_{i}}>\frac{2}{\xi_{i}}, \alpha<\mu \text {. }
$$

Accordingly at $\alpha \geq \mu$ we have a case of heavy fines. So,

$$
s_{i}^{\mathrm{OPT}}\left(\xi_{i}\right)=\left\{\begin{array}{cll}
\left((\alpha+\mu) / \alpha \xi_{i}\right)^{2} r_{i}, & \text { if } & \alpha<\mu ; \\
\left(2 / \xi_{i}\right)^{2} r_{i}, & \text { if } & \alpha \geq \mu .
\end{array}\right.
$$

Define the effect (including fines) of the winning enterprises:

$$
h_{i}\left(\xi_{i}\right)=\left\{\begin{array}{cll}
(\mu+\alpha)^{2} / \alpha \xi_{i} / r_{i}, & \text { if } & \alpha<\mu ; \\
\left(4 \mu / \xi_{i}\right) r_{i}, & \text { if } & \alpha \geq \mu .
\end{array}\right.
$$

Now from the equation $h_{i}\left(v_{i}\right)=\Delta_{i}$ it is possible to determine the marginal efficiency $v_{i}$ :

$$
v_{i}=\left\{\begin{array}{cll}
\frac{(\mu+\alpha)^{2}}{2 \alpha \mu} \sqrt{\frac{r_{i}}{c_{i}}}, & \text { if } & \alpha<\mu \\
2 \sqrt{\frac{r_{i}}{c_{i}}}, & \text { if } & \alpha \geq \mu .
\end{array}\right.
$$

Let enterprises be ordered in descending order $r_{i} / c_{i}: \frac{r_{1}}{c_{1}} \geq \frac{r_{2}}{c_{2}} \geq \Lambda \geq \frac{r_{n}}{c_{n}}$. 
Then the winners will be the first $m$ enterprises, and the reported effectiveness assessments in the equilibrium situation $\xi_{i}^{*}=v_{m+1}$.

\section{The optimality of the competitive mechanism}

Let's try to answer the question, how effective is the competitive mechanism for the distribution of limited resources. We denote by $R_{m}=\sum_{i=Q} s_{i}^{\mathrm{OPT}}\left(\xi_{i}\right)$. Consider the problem of optimal resource allocation $R_{m}$ between winners by the criterion of the maximum of the total effect. As is known, if the effect's functions are concave and differentiable, then a sufficient condition for the optimality of some solution $x^{*}$ is equality at the point $x^{*}$ of the derivatives $\frac{d \varphi_{i}\left(x_{i}^{*}\right)}{d x_{i}}=\lambda, i=1,2, \ldots, m$.

We now note that in the case of weak penalties, the optimal bids of the winners satisfy the conditions (4). And since for all the winners in an equilibrium situation $\xi_{i}^{*}=v_{m+1}$, then

$$
\frac{d \varphi_{i}\left(s_{i}^{\mathrm{OPT}}\right)}{d s_{i}}=\frac{\alpha v_{m+1}}{\alpha+\mu}, i=1,2, \ldots, m,
$$

resource $R_{m}$ is distributed among the winners in an optimal way. For the case of strong penalties, the optimal orders in equilibrium satisfy a different condition:

$$
\varphi_{i}\left(s_{i}\right)=v_{m+1} s_{i}^{O P T}
$$

from which in the general case does not follow the optimality of resource allocation between the winners. However, if the effect functions $\varphi_{i}\left(x_{i}\right)$ satisfy the system of differential equations

$$
\frac{d \varphi_{i}}{d x_{i}}=F\left[\frac{\varphi_{i}}{x_{i}}\right], i=1,2, \ldots, m
$$

where $\mathrm{F}$ - an arbitrary single-valued function (the same for all $i$ ), then in the case of heavy fines, the distribution of resources among the winners will be optimal. This fact follows directly from condition (12). Indeed, from (12) follows the constancy of the relation $\varphi_{i}\left(s_{i}^{O P T}\right) / s_{i}^{O P T}$ for all the winners in the equilibrium situation, and therefore, by (13), and the equality of the derivatives in equilibrium for all the winners. The results are formulated in the form of a theorem.

Theorem 2. (on the optimality of competitive mechanisms). In the case of weak penalties, the competitive mechanism ensures an optimal allocation of resources among the winners in the Nash equilibrium situation. In the case of heavy fines, the optimal allocation of resources between winners in Nash equilibrium is ensured if the winners function satisfies the system of differential equations (13).

Example 2. Consider the effect functions of the example 1:

$$
\varphi_{i}\left(x_{i}\right)=2 \sqrt{r_{i} x_{i}}, \quad i=1,2, \ldots, n
$$

We have: 


$$
\frac{d \varphi_{i}}{d \chi_{i}}=\sqrt{\frac{r_{i}}{x_{i}}}=\frac{\sqrt{r_{i} x_{i}}}{x_{i}}=\frac{\varphi_{i}}{2 x_{i}}, i=1,2, \ldots, n .
$$

Thus, the function of the effect of all enterprises satisfies the system (13), where F linear function. Therefore, for any set of winners, the distribution of resources between them will be optimal.

Theorem 2 can be used to find an approximate solution to the optimal resource allocation problem

$$
\begin{gathered}
\mathrm{F}(\mathrm{x})=\sum_{i=1}^{n} \varphi_{i}\left(x_{i}\right) \rightarrow \max _{\left\{x_{i}\right\}} \\
\sum_{i=1}^{n} x_{i} \leq R .
\end{gathered}
$$

Let us consider the case of weak fines.

If the quantity $\left[\sum_{i=m+1}^{n} \varphi_{i}\left(x_{i}\right)\right] /\left[\sum_{i=1}^{n} \varphi_{i}\left(x_{i}\right)\right]$, characterizing the "share" of the effect of losers in the competition of elements is small, then the approximate solution $\tilde{x}=\left\{\tilde{x}_{i}\right\}$ is determined by the expression

$$
\widetilde{x}_{i}= \begin{cases}\theta_{i}\left(v_{m+1}(\widetilde{c})\right), & \text { if } i \in \theta \\ \widetilde{c} & \text { if } i \notin \theta\end{cases}
$$

where the quantity $\widetilde{c}$ satisfies equation

$$
\sum_{i \in Q} \tilde{\chi}_{i}+(n-m) \tilde{c}=R
$$

Thus, the calculation of the approximate solution reduces to finding the value $\widetilde{c}$, at which (19). To find $\widetilde{c}$ you can build a variety of iterative schemes, in which the parameter $\widetilde{c}$ is selected depending on the sign of the value $\sum_{i=Q} \theta_{i}\left(v_{m+1}(\widetilde{c})\right)+(n-m) \widetilde{c}-R$. If this value is negative, the Center increases $\widetilde{c}$, if positive, then the center decreases $\widetilde{c}$.

When using such schemes, the value $\widetilde{c}$ depends on the strategies of consumers. Therefore, the equilibrium can differ from that which is established in Theorem 1. We give some considerations that enable us to estimate the equilibrium point obtained in this case $\left\{\xi_{i}^{*}\right\}$.

To determine strategies $\xi_{i}^{*}$ for $i \in Q$ notice, that $\theta_{i}\left(v_{m+1}\right)$-decreasing function $v_{m+1}$, therefore, the more $v_{m+1}$, the more $\tilde{c}$. Since the elements $i \notin Q$ are interested in increasing $\tilde{c}$, then at least the element with the number $m+1$ will report such an estimate $\xi_{m+1}$, at which $\widetilde{c}$ it would be possible to be large. As $\xi_{m+1} \leq v_{m}$, then in solving the game $\xi_{m+1}^{*}=v_{m}$. As for the remaining non-consumables, they can report any estimates $\xi_{i}<v_{m}$.

So, if the "weight" of the winners in the total mass of elements is sufficiently large, then the described competitive mechanism gives a close to the optimal allocation of resources. Obviously, for this purpose it is expedient to take as many winners as possible $\mathrm{m}=\mathrm{n}-1$. 


\section{Conclusion}

In this article, a competitive mechanism is investigated using the resource allocation problem as an example. Similar results could also be given for a more general model, where the active elements are not allocated resources, but different promotion functions, depending on whether the element is the winner in the competition or the loser. Such models were considered for the case when the elements are divided into only two groups: winners and losers $[7,8]$.

Note that competitive mechanisms are very close to competitive ones, in which winners are determined at the implementation stage based on the results of the work, while in the competitive mechanisms the winners are determined at the planning stage and have preferential terms for formation of plans. This seemingly insignificant difference leads to fundamental differences in the results of the analysis. So, in competition mechanisms, as a rule, there is no Nash equilibrium and we have to introduce more complex game decision definitions.

\section{References}

1. V.N. Burkov, B. Danaev, A.K. Enaleev, T.B. Naneva, D.P. Podval'niy, B.S. Yusupov, AandT 11, 142-153 (1988)

2. S.T. Rapatckaya, Mathematical modeling of competition mechanisms AandT, 4 (1987)

3. V.N. Burkov, Trends and management 4, 428-440 (2014)

4. V.L. Poryadina, Yu.V. Bondarenko, A.N. Chekomazov, Scientific and technical journal 1 (59), 20-24 (2015)

5. V.L. Poryadina, Management of socio-economic projects: a competitive approach: a monograph (Scientific Book, Voronezh, 2015)

6. A. Borboni, R. Faglia, Journal of Applied Mechanics, Transactions ASME 80(2), 021003 (2013) DOI: 10.1115/1.4007721

7. A. Borboni, M. Mor, R. Faglia, Journal of Dynamic Systems, Measurement and Control 138(11), 111003 (2016) DOI: $10.1115 / 1.4033831$

8. V. Kankhva, MATEC Web of Conferences 106, 08027 doi.org/10.1051/matecconf/201710608027 